\title{
Microscopy101
}

\section{Making Your Own Hair Probe: A Simple Guide}

\section{Bob Chiovetti}

RMC Products / Boeckeler Instruments, Tucson, AZ 85714

bob@boeckeler.com

\section{Introduction}

In our travels to meet with customers around the world, researchers often ask us how they can make their own hair probes. On an ultramicrotome, these hair probes are used to precisely manipulate thin sections of samples near the diamond knife while avoiding damage to the knife.

You might choose to make your own hair probe to get exactly the type of handle or hair that works best in your application. For example, when working with cryo-ultramicrotomes, you would need a longer handle than for room-temperature probes so that you can reach down into the cryo-chamber without getting frostbite. Making your own hair probe can also be less expensive than purchasing the commercial probes, given that a single eyelash on a handle can cost between $\$ 12$ and $\$ 35$.

Over the years, we've heard many clever ideas on hair probe construction methods. We share one here that's very simple.

\section{Materials}

Although ultramicrotomists across the globe have their own preferences for which material to use for the handle, from (a)

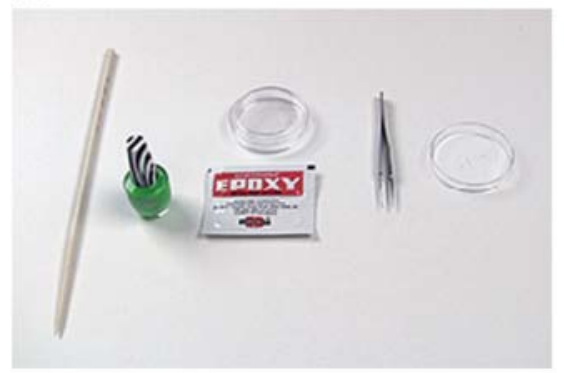

(c) (b)

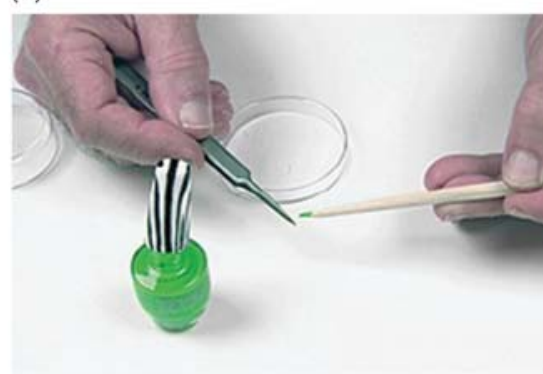

(d)

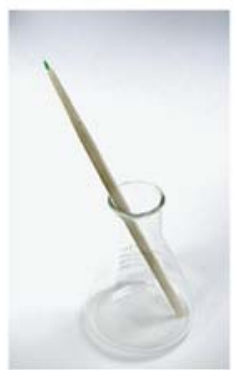

Figure 1: (a) Tools needed to make this hair probe, including (left to right) a wooden chopstick, nail polish, epoxy and a plastic petri dish, a pair of fine forceps, and a petri dish with individual eyelash hairs (or Dalmatian hairs). (b) Attaching the eyelash to the pointed end of the chopstick, which has been painted with nail polish. (c) Close-up of the eyelash attached to the chopstick. (d) Completed eyelash probe drying upright for about 30 minutes. plastic drinking straws to orangewood sticks and chopsticks, we've found that many people prefer bamboo chopsticks. They are not only the right length to use in the cryo-ultramicrotome, but their beveled edges make them easier to hold firmly, and when you set them down on the table, they don't roll, as a straw or orangewood stick might.

As to the type of hair selected, many prefer a single human eyelash or a Dalmatian dog hair. The latter is excellent because Dalmatian hairs taper to an extremely fine point and have a tougher flex than human eyelashes. (Plus you get to keep your eyelashes intact!)

\section{Setup}

The tools needed to make our simple hair probes include the following:

- a pencil sharpener (optional)

- a bamboo chopstick, which is our preference, but you can use any slim wooden handle about 6 to 8 inches long.

- a bottle of nail polish or epoxy quick-set cement that is mixed on aluminum foil or in a plastic petri dish

- hairs (Dalmatian hair or human eyelashes)

- a pair of fine forceps to manipulate the eyelashes

\section{Procedure}

1. You can use a pencil sharpener to make a more pointed tip on the chopstick.

2. Apply a small amount of nail polish or freshly mixed epoxy glue to the end of the chopstick (see Figure 1b).

3. Using the forceps, place the root of the hair in the epoxy or nail polish, then place it at the end of the chopstick, with the tip of the hair projecting outward.

Note: You may want to do this under the stereomicroscope so that you can find the proper end of the hair and position it accurately on the end of the chopstick.

4. Let the chopstick dry about 30 minutes

You are now ready to use your new hair probe.

For a 10-minute video detailing these steps, as well as steps for constructing other types of hair probes and loops used in ultramicrotomy, see https://www.youtube.com/watch?v=x_vkf b6e_WM. 


\section{MICROSCOPES \\ N}

\section{Isn't it about time you had your own Digital Microscope?}

Now you can with the portable, affordable uScopeMXII!

The uScopeMXII is a small digital desktop microscope you can use in your workplace or home office. It captures images from standard glass slides and sends them to your PC.

You can interactively browse slides with full control of focus, image processing, and location. You can also scan regions of interest creating fully focused image sets.

The industry-standard USB interface makes it simple to plug in and start capturing images. It easily interfaces with your desktop or laptop PC and allows you to view and capture slide images in a wide variety of environments.

The uScopeMXII is manufactured in the United States.

\section{Features and Benefits}

- Overview and Objective Cameras The uScopeMXII has an objective camera for scanning and an overview camera for navigating.

- Automatic Focus

Images are automatically focused using configurable focus algorithms.

- Portability

At a weight of about $5 \mathrm{lbs}$., the uScopeMXII is highly portable.

- Easy to Use

The uScope Navigator software simplifies scanning and browsing.

\section{- Self-Contained}

The uScopeMXII is self-contained and includes the electronics, cameras, stage, and optics in a device about the size of a large external disk drive.

\section{- Full Imaging Control}

User filters provide complete control over image processing and correction.

$$
\text { . }
$$

